\title{
Differenzialdiagnose benigner, multifokaler, nicht infektiöser Leberläsionen
}

\section{Differentials of Benign, Multilocular, Non-Infectious Liver Lesions: An Imaging Approach}

\section{Einleitung}

Benigne Leberläsionen sind klinisch meistens asymptomatisch und fallen erst als Zufallsbefund, z. B. im Ultraschall bei Routineuntersuchungen, auf. Diagnostisch entscheidend ist ihre bildmorphologische Abgrenzung zu malignen Raumforderungen der Leber, die bei Erwachsenen mit Abstand häufiger anzutreffen sind. Kenntnisse über deren Existenz, Häufigkeit und insbesondere über deren Erscheinungsmuster in der Bildgebung sind neben Anamnese, Klinik und laborchemischen Ergebnissen daher von hoher Bedeutung.

\section{Hepatische Adenomatose}

Die hepatische Adenomatose (HA), erstmals 1985 durch Flejou et al. beschrieben, ist charakterisiert durch das Vorliegen von mindestens 10 Adenomen in einem sonst unauffälligen Leberparenchym. Sie wird zumeist in der vierten Lebensdekade bei einem mittleren Alter von 32 Jahren diagnostiziert und ist mit einer Mutation der Gene HNF1A sowie MODY Typ 3 assoziiert. Hepatozelluläre Adenome (HCA) lassen sich histopathologisch in drei Subtypen einteilen, das inflammatorische HCA, das HNF- $1 \alpha-H C A$ und das $\beta$-Catenin-aktivierte $\mathrm{HCA}$, welche sich biologisch unterschiedlich verhalten. Die HA ist meistens nicht mit der Pilleneinnahme assoziiert und daher anamnestisch schwieriger einzuordnen (Dharmana $\mathrm{H}$ et al. Clin Radiol 2016; 72: 276 - 285).

Bildgebung: Sonografisch sind die Läsionen scharf begrenzt, nicht lobuliert und können Einblutungen, Nekrosematerial und Fett enthalten. Der zumeist hohe Fettgehalt stellt sich hyperechogen dar. Die CT ermöglicht eine differenziertere Diagnostik. Abhängig vom Fettgehalt zeigen sich im Nativbild hypo- bis isodense Läsionen. In der arteriellen Phase zeigt sich zumeist ein homogenes Kontastmittelenhancement, während sich in der portalvenösen Phase neben intraläsionalen arteriovenösen Kontastmittelshunts ein schnelles
KM-Auswaschen zeigt. Je größer die Adenome sind, desto heterogener wird ihr $\mathrm{Er}$ scheinungsbild. Aufgrund deren zahlreichen Erscheinens fällt die Differenzierung zu Lebermetastasen schwer. Die MRT eignet sich insbesondere zur Subtypisierung. I-HCA zeigen in der T2-Wichtung typischerweise ein heterogenes Signalverhalten mit einem stärkeren Signal in der Peripherie der Läsionen („atoll-sign“). Typischerweise persistiert das arterielle Enhancement bis in die portalvenöse Phase (Grazioli L et al. Radiology 2000; 216: 395 - 402). Akute intraläsionale Einblutungen sind hyperintens in T1, während chronische Einblutungen in T1 und T2 eher hypointens zur Darstellung kommen. HNF$1 \alpha$-HCA zeigen ebenso ein vermehrtes Kontrastmittelenhancement in der arteriellen Phase, welches jedoch nicht bis in die portalvenöse Phase persistiert. Mittels Einsatz des MR-Kontrastmittels Gadoxetsäure (Primovist ${ }^{\circledR}$ ) lassen sich mit hoher Sensitivität und Spezifität HCA von FNHs unterscheiden ( $\vee$ Abb. 1a, b). Die meisten FNHs zeigen in der hepatobiliären Phase eine unveränderte oder hohe Signalintensität, während HCA typischerweise hypointens im Verhältnis zum übrigen Leberparenchym erscheinen (Grazioli L et al. Radiology 2000; 216: 395 -402).
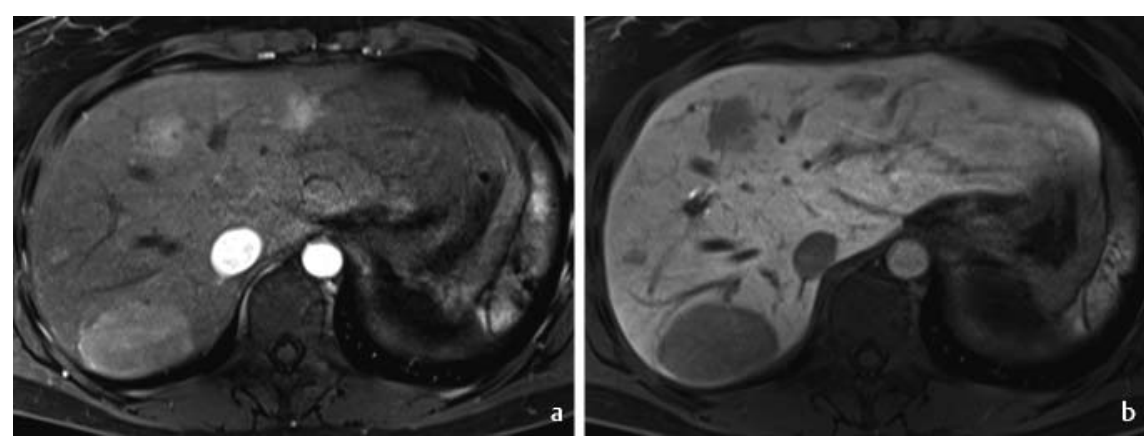

- Abb. 1 MRT der Leber eines Patienten mit hepatischer Adenomatose. a In der früharteriellen Phase post Gadoliniumgabe zeigen sich in der T1-fettgesättigten Sequenz hyperintense Leberläsionen. b In der späten (hepatobiliären) T1-fettgesättigten Sequenz kommen die Läsionen als Aussparungen im KM-hyperintensen Lebergewebe zur Darstellung, was einem wichtigen Differenzierungsmerkmal zur FNH entspricht. 

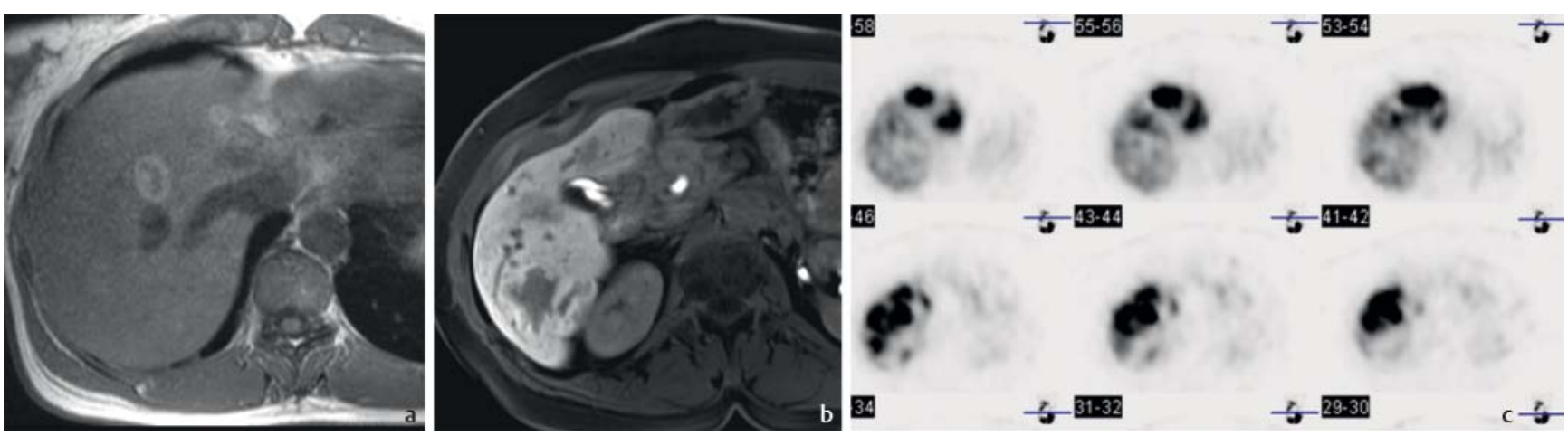

- Abb. 2 MRT und 99mTc-HIDA-Szintigrafie der Leber eines Patienten mit follikulärem nodulären Hyperplasiesyndrom. a In der nativen T1-Sequenz stellen sich teils hyperintense Läsionen dar, welche in der $\mathbf{b}$ späten (hepatobiliären), T1-fettgesättigten Sequenz post Gadoliniumgabe FNH-typische Randanreicherungen zeigen. c In der 99mTc-HIDA-Szintigrafie lassen sich multifokale Anreicherungen.

hyperintens in T2. Sog. nicht klassische FNHs, wie z. B. die teleangiektatische Form, können atypische Bildcharakteristika aufweisen ( $\mathbf{A} \mathbf{A b} \mathbf{b} . \mathbf{2 a}-\mathbf{c}$ ) (Anderson SW et al. RadioGraphics 2009; 29: 211 229). Hämangiome im Rahmen des FNHSyndroms verhalten sich dagegen meistens typisch.

\section{(Multi)fokale Steatosis hepatis}

Hepatische Steatosen haben meistens eine diffuse oder landkartenähnliche Verteilung, selten können sie aber multifokal („steatosis areata“) auftreten und daher anderen hepatischen Läsionen ähneln. Die Abgrenzung gestaltet sich besonders im Ultraschall und in der CT schwierig. Aufschluss können die Lokalisationen der Läsionen in der Leber liefern. Fokale Steatosen finden sich häufig im Bereich des Gallenblasenbettes, des falciformen Ligaments und der Leberpforte. Sie verdrängen mit ihrer Masse keine benachbarten Strukturen wie Gefäße. Schwierig wird deren Differenzierung von z. B. Lebermetastasen, wenn sie einen nodulären Charakter aufweisen. Alternativ finden sich fokale, nicht steatotische Areale (z. T. mit nodulärem Charakter) bei Patienten mit diffuser Leberverfettung (Jang JK et al. Abdom Radiol 2017; 42: 1667 -1678).

Bildgebung: Sonografisch lassen sich scharf umschriebene, hyperechogene Areale abgrenzen. In der CT erscheinen sie hypodens ( $\boldsymbol{A} \mathbf{A b} \mathbf{b} . \mathbf{3 a}$ ). Eine Differenzierung zwischen der multifokalen Steatose und der HA kann in der MRT mittels In-phase und Opposed-phase T1-gewichteten Sequenzen erfolgen ( $\mathbf{A} \mathbf{b} \mathbf{b} . \mathbf{3 b}$ ).
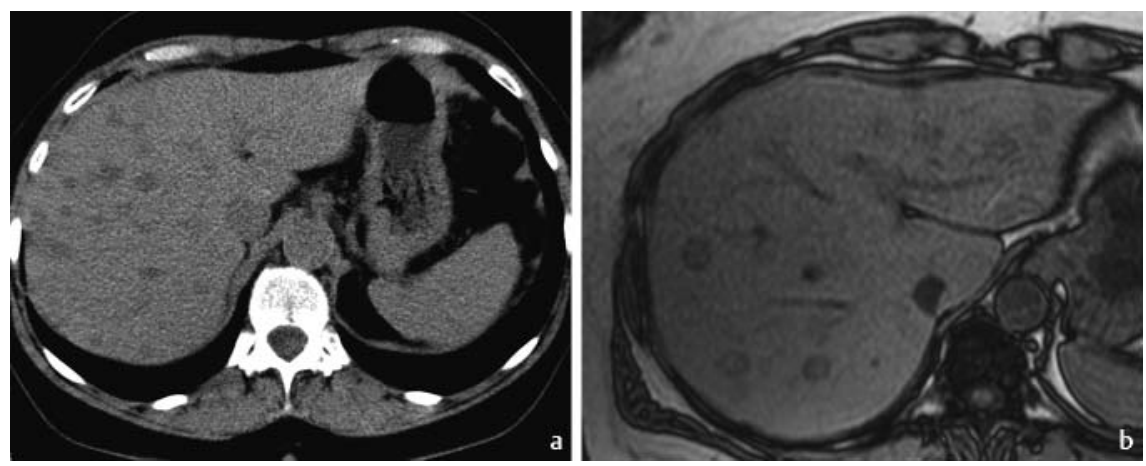

- Abb. 3 a In der nativen CT der Leber eines Patienten mit multifokaler Steatose zeigen sich homogen hypodense Läsionen, welche b MR-morphologisch in der nativen T1-Sequenz teils Signalauslöschungen zeigen.

Multifokale Steatosen zeigen in der Opposed-phase Signalauslöschungen. Noch seltener können fokal-noduläre Verfettungen eine Schießscheibenkonfiguration annehmen. Ein konträres Verhalten wird im Falle fokaler, nicht steatotischer Leberareale unter Nutzung derselben Sequenzen erwartet (Jang JK et al. Abdom Radiol 2017; 42: $1667-1678$, Anderson SW et al. RadioGraphics 2009; 29: 211 - 229).

\section{Noduläre regenerative Leber- hyperplasie}

Noduläre regenerative Hyperplasien werden oft post mortem in Autopsien entdeckt. Sie bestehen aus einer Transformation des normalen Leberparenchyms in hyperplastische regenerative Knoten, welche in ihrer Größe zwischenmikroskopisch variieren und bei mehreren Zentimetern tumorähnlich imponieren können. Die fehlende Fibrose unterscheidet sie von der
Leberzirrhose. Sie entsteht oft sekundär im Rahmen systemischer Erkrankungen, wie z. B. myeloproliferativer oder immunologischer Erkrankungen (Dachman $\mathrm{AH}$ et al. AJR 1986; 148: 717 - 722).

Bildgebung: CT-morphologisch sind die Knoten typischerweise hypodens und zeigen nur ein dezentes Enhancement nach Kontrastmittelgabe, insbesondere in den peripheren Randkonturen der Knoten. MR-morphologisch können die Knoten sowohl hypo-, iso- als auch hyperintens erscheinen ( $\mathbf{A} \mathbf{b b} \mathbf{4} \mathbf{4 a}, \mathbf{b})$. In der T2-Wichtung können sie einen Halo und in der T1Wichtung ein peripheres Kontrastmittelenhancement aufweisen (Anderson SW et al. RadioGraphics 2009; 29: 211 - 229).

\section{Leberhämangiomatose}

Hämangiome sind vaskulären Ursprungs und stellen die häufigsten benignen Lebertumore dar. Sie treten sowohl solitär als 

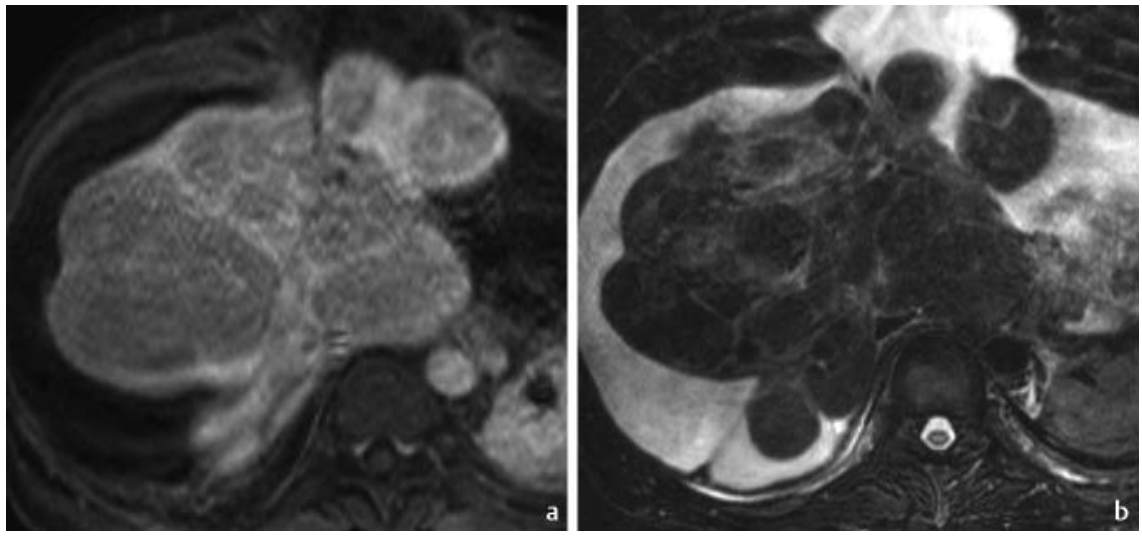

- Abb. 4 MRT der Leber eines Patienten mit nodulärer regenerativer Hyperplasie. a In der T1 fettgesättigten Sequenz post Gadoliniumgabe kommen multiple hypointense, irregulär konfigurierte Leberläsionen zur Darstellung. Das umgebende Leberparenchym ist zirrhotisch verändert und zeigt multiple Kapseleinziehungen. b In der T2-fettgesättigten Sequenz erscheinen die intrahepatischen Läsionen hypointens und teils septiert, das perihepatische Fettgewebe stellt sich hyperintens dar.

auch multipel auf (Elsayes KM et al. AJR 2008; 190: 1291 - 1299).

Bildgebung: Sonografisch stellen sich Hämangiome als fokale, homogene und hyperechogene Läsionen dar. In Abhängigkeit des Fettgehaltes einer Leberfibrose variiert das Erscheinungsbild. Im nativen
CT sind die Läsionen typischerweise hypodens. Nach Kontrastmittelgabe zeigen sie im typischen Fall in der Spätphase ein zentripetales Enhancement. In der arteriellen Phase können Hämangiome abgängig von der Größe teilweise oder vollständig aufhellen. Interessanterweise verhalten sich multiple Hämangiome eher atypisch mit hypoechogenem US-Erscheinen und rascher KM-Aufnahme (kein IrisblendenPhänomen). Alternativ zeigen sie eine primär zentrale KM-Aufnahme („dot sign“) oder gar ein zentrifugales Enhancement (,inside-out“) ( $\mathbf{A b b . 5 a - c ) ~ ( K i m ~ S ~ A J R ~}$ 1999; 174: 1571 - 1574, Elsayes KM et al. AJR 2008; 190: 1291 - 1299).

\section{Multifokale Peliosis hepatis}

Die Peliosis hepatis ist eine seltene Erkrankung, charakterisiert durch blutgefüllte Zysten in der Leber und Dilatationen von Sinusoiden. Es werden medikamentös-toxische, infektiöse, malignitätsbedingte und diabetesassoziierte Genesen neben idiopathischen Fällen beschrieben (Kim SH et al. J Comput Assist Tomogr 2007; 31: 79-85).

Bildgebung: Im Ultraschall zeigen sich je nach Leberfettgehalt hypo- oder hyperechogene Läsionen. Duplexsonografisch können Gefäße in den Läsionen sichtbar werden. Im nativen CT zeigen sich fokale hypodense Areale, welche durch Einblu- 

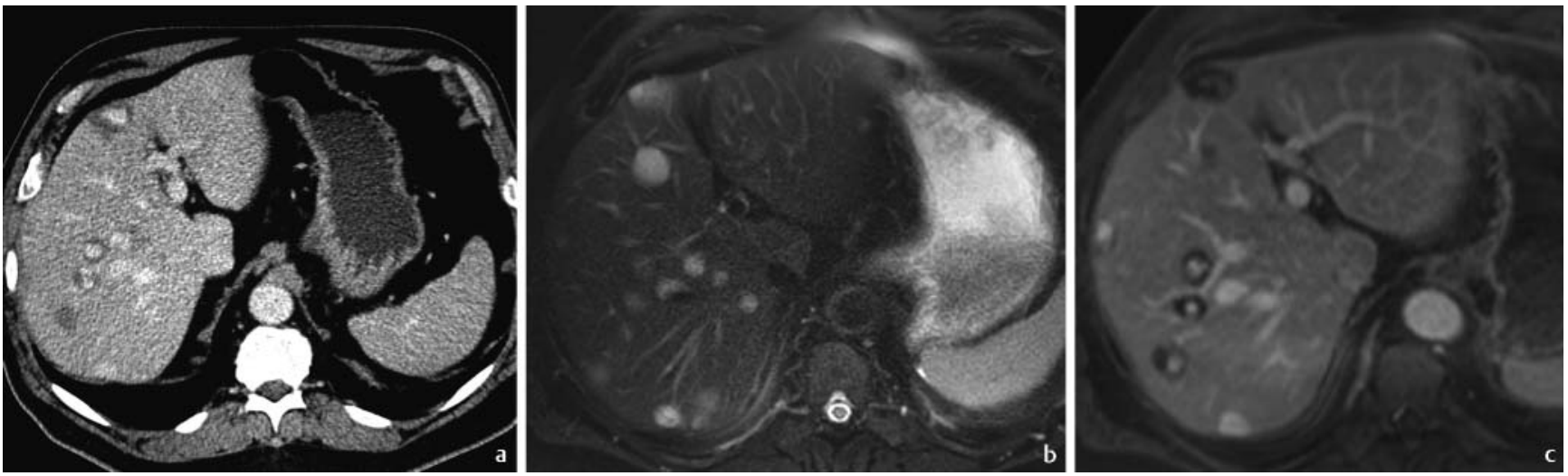

- Abb.5 CT und MRT der Leber eines Patienten mit Hämangiomatose und atypischem, zentrifugalen KM-Enhancement („inside-out“). a In der CT kommen nach i. v.-Applikation von jodhaltigem KM in der portalvenösen Phase hypodense Läsionen mit zentralem Enhancement zur Darstellung. $\mathbf{b}$ MR-morphologisch erscheinen in der nativen T2-fettgesättigten Sequenz die Läsionen homogen hyperintens, während sich in der c T1-fettgesättigten Sequenz post Gadoliniumgabe hypointense Läsionen mit zentral hyperintensem Enhancement zeigen.

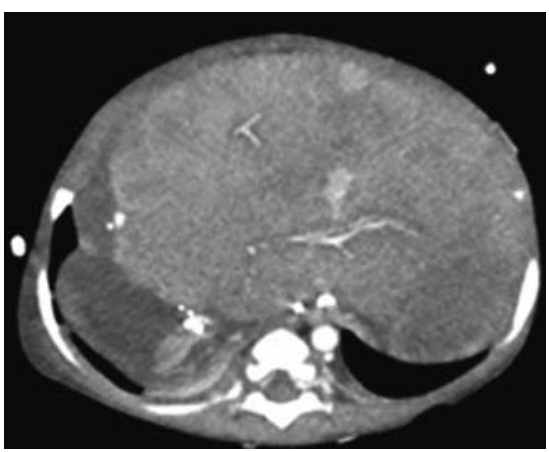

- Abb. 6 CT der Leber eines Patienten mit Peliosis hepatis. Nach i. v.-Gabe von jodhaltigem Kontrastmittel zeigen sich in der arteriellen Phase zwei intrahepatische Läsionen mit homogenem KM-Enhancement.

tungen noch hypodenser erscheinen können. Nach Kontrastmittelapplikation zeigen die Areale in der arteriellen Phase ein niedriges Enhancement im Vergleich zum übrigen Lebergewebe und reichern bis zur portalvenösen Phase zentripetal oder zentrifugal Kontrastmittel an ( $\triangleright$ Abb.6). In der Spätphase sind die Läsionen typischerweise homogen hyperdens (Kim SH et al. J Comput Assist Tomogr 2007; 31: 79 - 85).

\section{Budd-Chiari-Syndrom assozi- ierte Leberläsionen}

Das Budd-Chiari-Syndrom ist charakterisiert durch eine primäre oder sekundäre, lobuläre oder segmentale Lebervenenobstruktion. Zu den primären Ursachen gehören angeborene Fehlbildungen oder

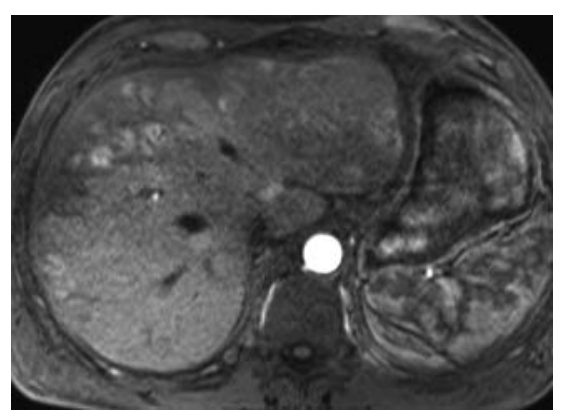

- Abb. 7 MRT der Leber eines Patienten mit inkomplettem Budd-Chiari-Syndrom. In der T1-fettgesättigten Sequenz post Gadoliniumgabe zeigt sich in der arteriellen Phase ein Leberparenchym mit heterogenem Enhancement und multiplen, hyperintensen intrahepatischen Läsionen.

traumatische Verletzungen, sekundäre Ursachen sind oftmals thrombotische Verschlüsse (Torabi M et al. RadioGraphics 2008; 28: 1967 - 1982).

Bildgebung: Bei chronischen Verläufen sind zudem oftmals Kollateralkreisläufe ausgebildet, die Leber zeigt ein eher heterogenes Kontrastmittelenhancement und gelegentlich zeigen sich auch multiple vaskuläre Knoten ( $\triangleright$ Abb. 7). Diese repräsentieren regenerative Knoten mit kräftiger und homogener KM-Aufnahme in der arteriellen Phase und Persistenz derselben in der portalvenösen Phase, welches eine Differenzierung zum hepatozellulären Karzinom ermöglicht (Torabi $\mathrm{M}$ et al. RadioGraphics 2008; 28: 1967 -1982).

\section{Fokale atypische Hämochro-} matose

Eine vermehrte Ablagerung von Eisen in der Leber wird bei vielen Erkrankungen, wie die hereditäre Hämochromatose, Leberzirrhose, hämolytische Anämie oder im Zusammenhang mit Bluttransfusionen beschrieben. In der Regel verteilt sich das Eisen diffus im Leberparenchym oder erscheint etwas betont innerhalb von zirrhotischen Regeneratknoten (Horger et al. Rofo 2006; 178(7): 657-660).

Bildgebung: In seltenen Fällen können die Eisenablagerungen in der Leber inhomogen ausfallen, sodass ein multifokales Tumorgeschehen vorgetäuscht werden kann ( $\mathbf{A b b} \mathbf{8} \mathbf{8 a}-\mathbf{c}$ ). Das sicherste Verfahren zur Diagnosesicherung ist die Nutzung von T2-gewichteten Sequenzen, die zugleich auch eine Eisenquantifizierung zulassen. In der nativen CT kann sich die Leber bei höhergradiger Hämochromatose dichter (> $75 \mathrm{HU}$ ) darstellen und somit ebenfalls diagnostiziert werden (Brittenham GM, Badman DG. Blood 2003; 101: $15-19)$.

\section{Hepatisches Sinusobstrukti- onssyndrom (HSOS)}

Das HSOS ist ein lebensbedrohliches Krankheitsbild, welches mit einer schmerzhaften Hepatomegalie, Aszites und Hyperbilirubinämie einhergeht. Als Auslöser werden u. a. Hochdosis-Chemotherapie, Bestrahlung und pflanzliche Medikamente be- 

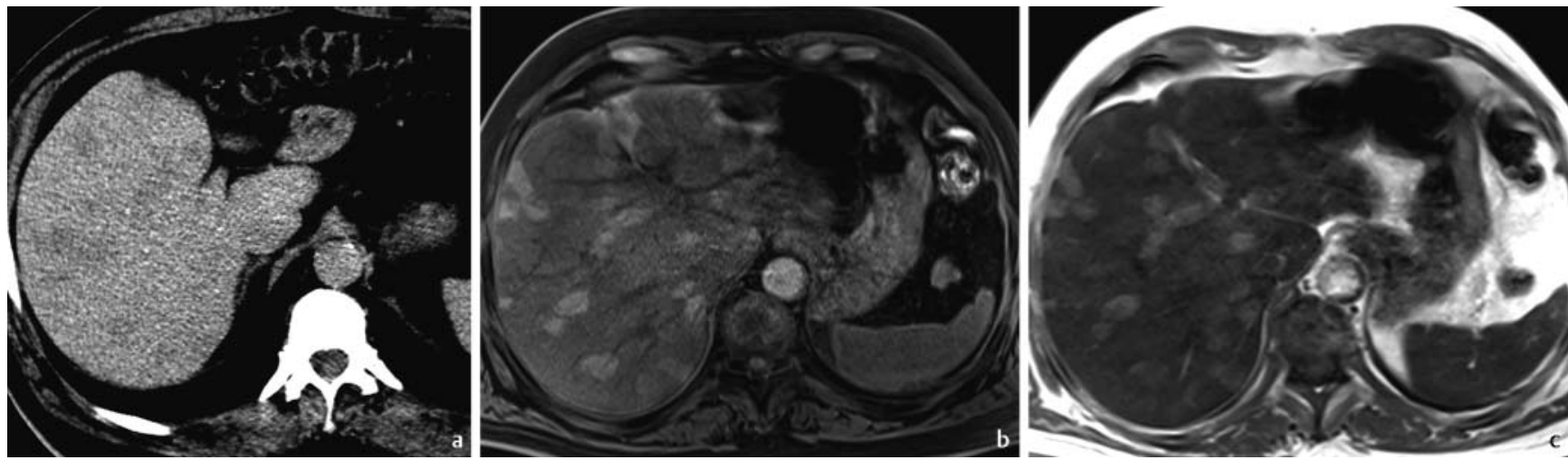

- Abb. 8 CT und MRT der Leber eines Patienten mit Hämochromatose. a In der nativen CT zeigen sich multiple hypodense Leberläsionen mit Dichtewerten > 100 HU. In der b T1-gewichteten MRT kommt ein multinoduläres Muster hyperintenser Läsionen zur Darstellung. Diese sind auch in der c T2-gewichteten Sequenz nachvollziebar. Die Läsionen entsprechen fokalen, nicht hämosiderotischen Arealen.
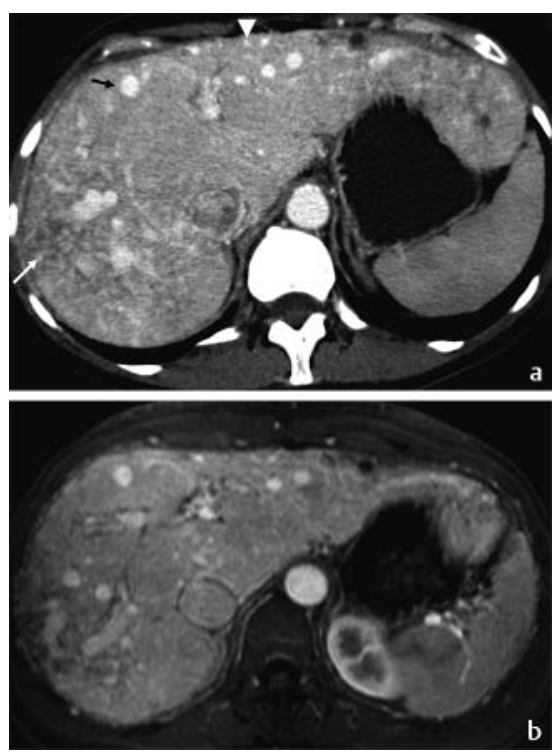

Abb. 9 CT und MRT eines Patienten mit hereditärer hämorrhagischer Teleangiektasie (Osler-Weber-Rendu-Syndrom). a In der CT kommen nach i. v.-Applikation von jodhaltigem KM in der früharteriellen Phase multiple, z. T. subkapsulär lokalisierte, hypervaskularisierte Läsionen (schwarzer Pfeil und Pfeilspitze) mit benachbarten Regeneratknoten und AVShunts (weißer Pfeil) zur Darstellung. b In der MRT zeigen sich die Läsionen in der portalvenösen Phase post Gadoliniumgabe in der T1-fettgesättigten Sequenz hyperintens.

schrieben (Zhou H et al. Korean J Radiol 2014; 15: 218 - 225).

Bildgebung: Bildmorphologisch zeigen sich nach Kontrastmittelapplikation vor allem ein fleckiges Enhancement und Aszites. Zudem zeigen sich eine Hepatomegalie, eine Gallenblasenwandverdickung
(>3 mm) und eine Lebervenenverengung. In der MRT finden sich in T2 hohe Signalintensitäten im Portalvenen umgebenden Parenchym im Sinne eines periportalen Manschettenzeichens. Das den Lebervenenstern umgebende, gesunde Leberparenchym zeigt in der venösen Kontrastmittelphase typischerweise ein physiologisches Enhancement. Dieses hebt sich daher von dem fleckförmigen, reduzierten Enhancement der betroffenen Leberareale deutlich ab, was ein kleeblattartiges Aussehen hervorrufen kann (Zhou H et al. Korean J Radiol 2014; 15: 218 - 225). Manchmal können allerdings multifokale Nodularitäten nach i. v.-KM-Gabe zur Darstellung kommen. Diese können Metastasen vortäuschen.

\section{Hereditäre hämorrhagische Teleangiektasie (Osler- Weber-Rendu-Syndrom)}

Die hereditäre hämorrhagische Teleangiektasie ist eine autosomal dominante Multiorgankrankheit, welche mit fibrovaskulären Dysplasien sowie multiplen Teleangiektasien einhergeht und von arteriovenösen Malformationen begleitet wird. In der kontrastmittelgestützten CT zeigt die Leber ein heterogenes Enhancement mit multiplen Arealen kurzzeitigen Enhancements, bedingt durch das Vorliegen arterioportaler Shunts, Teleangiektasien und konfluierender Gefäße. Dabei entstehen auch stark vaskularisierte, noduläre Leberläsionen, die Metastasen bzw. hepatozelluläre Karzinome vortäuschen können
( Abb. 9a, b) (lanora AA et al. Radiology 2004; 230: 250 - 259).

\section{Inflammatorische Pseudotu- moren}

Inflammatorische Pseudotumoren bestehen aus Entzündungszellen und fibrösem Bindegewebe. Sie können sporadisch oder sekundär im Rahmen einer Hepatitis auftreten. Sonografisch zeigen sich sowohl hypo- als auch hyperechogene Läsionen, welche septiert sein können. Im nativen CT sind die Läsionen hypodens, nach Kontrastmittelgabe kommt es typischerweise zu einem peripheren Enhancement oder zu einem Enhancement der Septen. In der MRT können sich die Läsionen verschieden darstellen, zumeist sind sie hypointens in T1 und hyperintens in T2 (Yan FH et al. World J Gastroenterol 2001; 7: 422 -424).

\section{Chemotherapie-induzierte} Hepatopathie durch fokale

\section{Pseudometastasen}

Durch Einsatz von Chemotherapeutika wie bspw. Oxaliplatin im Rahmen des FOLFOXSchemas zur adjuvanten Therapie kolorektaler Karzinome wird die Entstehung verschiedener fokaler Hepatopathien begünstigt, wie z. B. die Dilatation von Sinusoiden im Rahmen eines Chemotherapieassoziierten sinusoidalen Obstruktionssyndroms durch perisinusoidale Fibrose (You S et al. Korean J Radiol 2017; 18: 413 -426). Pathophysiologisch liegt der 

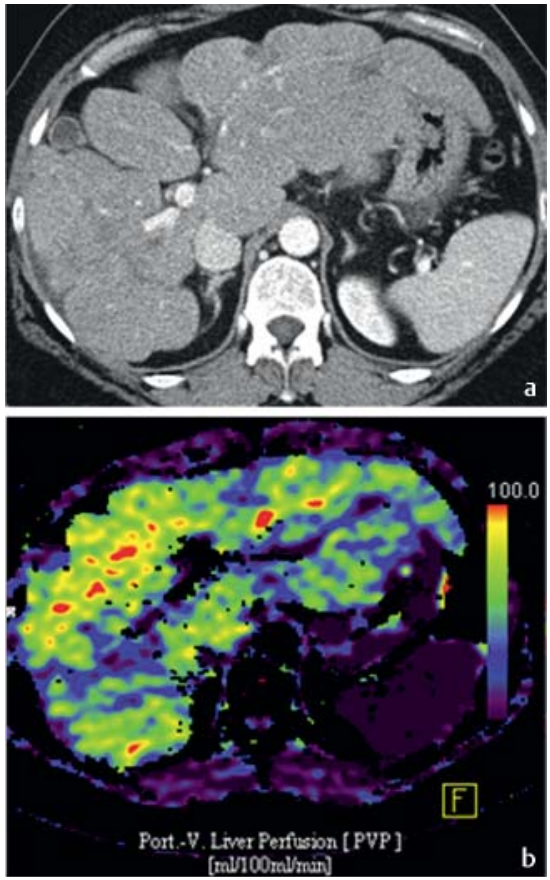

- Abb. 10 CECT der Leber a sowie Perfusions-CT (portalvenöse Perfusionsfarbkarte) der Leber $\mathbf{b}$ einer Patientin mit Mammakarzinom und erfolgreich behandelter Lebermetastasierung. Grobnoduläres, heterogenes Dichtemuster der Leber infolge der Metastasenrückbildung . Die Perfusions-CT zeigt ein überwiegend portalvenöses Perfusionsmuster der Leber bei fehlender portaler Hypertension, konträr zur Leberzirrhose.

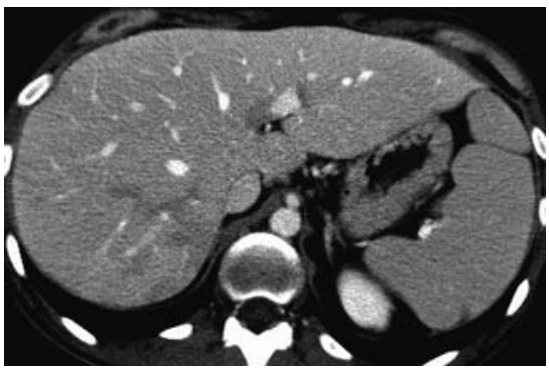

- Abb.11 CECT der Leber. Es zeigen sich multifokale Densitäten, hautsächlich im rechten Leberlappen, histologisch eosinophilen Granulomen entsprechend. Der Patient leidet an einem malignen Lymphom, unter laufender Chemotherapie ist es zu dieser Komplikation gekommen. perisinusoidalen Fibrose eine Schädigung sinusoidaler Endothelzellen zugrunde mit konsekutiver Aufhebung der Sinuswandintegrität.

Bildgebung: CT-morphologisch zeigen sich in der portalvenösen Kontrastmittelphase bei multifokaler Manifestation der Läsionen multifokale Hypodensitäten. MRmorphologisch charakteristisch sind im Gegensatz zu Metastasen eine unscharfe Begrenzung der Läsionen, keine kugelige Form sowie ein gemischtes Signalmuster (You S et al. Korean J Radiol 2017; 18: $413-426)$.

\section{Pseudozirrhose nach erfolg- reicher Therapie hepatischer Metastasen}

Eine multifokale Retraktion der Leberkapsel mit fokaler, sekundärer Parenchymhyperplasie wird im Fall von unter Therapie schrumpfenden Lebermetastasen (z.B beim Mamma-CA) beobachtet ( $\triangleright$ Abb. 10a, b). Die histopathologische und pathophysiologische Basis dieser Veränderungen ist wenig verstanden, daher ist die Bezeichnung "Pseudo-" unzutreffend, zumal diese Patienten eine portale Hypertension entwickeln können. In der Bildgebung wie auch histopathologisch unterscheiden sich diese Knoten nicht vom normalen Leberparenchym. Dennoch können sie aufgrund von Verdrängung und manchmal klarer Abgrenzung vom Leberparenchym wie Raumforderungen imponieren (Anderson SW et al. RadioGraphics 2009; 29: 211 - 229).

\section{Tumor-induzierte eosinophile Lebergranulome/Nekrosen}

Hepatische eosinophile Granulome/Nekrosen sind zumeist Folge parasitärer Infektionen. Jedoch können auch Tumore zur Entstehung eosinophiler Nekrosen führen, welche hepatischen Metastasen ähneln können ( $>$ Abb. 11). Die durch den
Primärtumor produzierten Cytokine IL-2, IL-5 und G-CSF gelangen über die Pfortader in die Leber und führen ihrerseits über eine Stimulation des eosinophilen chemotaktischen Faktors zur Aggregation eosinophiler Granulozyten. Bildmorphologisch zeigt sich typischerweise eine zentrale Nekrose.

\section{Weitere benigne multifokale Leberläsionen}

Dazu zählen die Lipomatose, die Angiomyolipomatose sowie die biliäre Papillomatose und die biliären Zystadenome. Des Weiteren können granulomatöse Erkrankungen (z. B. Sarkoidose) ein ähnliches Bild hervorrufen.

\section{Autoren}

Christian Philipp Reinert, Omar M. Albtoush, Michael Bitzer, Marius Horger,

Sorin Dumitru loanoviciu

\section{Abteilung Radiologie, Universitätsklinikum} Tübingen

\section{Korrespondenzadresse}

Prof. Marius Horger

Abteilung Radiologie

Universitätsklinikum Tübingen

Hoppe-Seyler-Str. 3

72076 Tübingen

Marius.Horger@med.uni-tuebingen.de

\section{Bibliografie}

DOI https://doi.org/10.1055/s-0043-113134 Online-Publikation: 2017 | Fortschr Röntgenstr 2017; 189: 921-926 @ Georg Thieme Verlag KG Stuttgart · New York ISSN 1438-9029 